\title{
Short-Term Teriparatide for Bone Marrow Edema Secondary to Complex Regional Pain Syndrome: Case Reports on Efficacy After Two Years of Follow-Up
}

\author{
Felice Galluccio ${ }^{1}$, Abdallah El Sayed Allam ${ }^{2}$, Francesco Perdisa ${ }^{3}$, Ke-Vin Chang ${ }^{4}$ \\ 1. Rheumatology - Experimental and Clinical Medicine, University of Florence, Florence, ITA 2. Physical Medicine, \\ Rheumatology and Rehabilitation, Tanta University Hospitals \& Faculty of Medicine, Tanta University, Tanta, EGY 3. \\ Hip and Knee Replacement, IRCCS Istituto Ortopedico Rizzoli, Bologna, ITA 4. Physical Medicine and Rehabilitation, \\ National Taiwan University Hospital, Bei-Hu Branch, Taipei, TWN
}

Corresponding author: Felice Galluccio, felicegalluccio@gmail.com

\begin{abstract}
Bone marrow edema secondary to chronic pain syndrome after knee trauma is a disabling condition that presents with localized pain, allodynia, edema, decreased range of motion and osteopenia. The management includes a variety of medications and rehabilitation. The treatment of refractory diseases is challenging for most physicians. We present two cases of refractory bone edema secondary to complex regional pain syndrome that were successfully treated with a short-term regimen of teriparatide (TPT), a biosynthetic analogue (PTH 1-34) of the human parathormone. The rapid and sustained pain reduction with recovery of knee function for two years following administration of TPT demonstrated its potential for the treatment of bone edema due to complex regional pain syndrome.
\end{abstract}

Received 04/27/2020

Review began 05/05/2020 Review ended 05/07/2020 Published 05/14/2020

\section{() Copyright 2020}

Galluccio et al. This is an open access article distributed under the terms of the Creative Commons Attribution License CC-BY 4.0., which permits unrestricted use, distribution, and reproduction in any medium, provided the original author and source are credited.
Categories: Pain Management, Orthopedics, Rheumatology

Keywords: bone marrow edema, complex regional pain syndrome, knee injury, teriparatide

\section{Introduction}

Bone marrow edema (BME) is a generic term used to describe a common finding on magnetic resonance imaging (MRI) and can either present spontaneously (Spontaneous osteonecrosis, insufficiency fractures, etc.) or originate from various pathologies (secondary BME, sBME) [1]. Among the traumatic causes of sBME, complex regional pain syndrome (CRPS) is probably the most disabling, characterized by regional pain with allodynia or hyperalgesia, edema, vasomotor instability, movement disorders, joint stiffness, and regional osteopenia [2,3]. The diagnosis of CRPS is mainly clinical and is defined as continuous pain disproportional to the triggering trauma with the associated clinical signs (temperature asymmetry, skin color changes or trophic changes, edema and sweating, muscular weakness, tremor or dystonia) based on the Budapest criteria [4]. Despite different therapeutic approaches (corticosteroids, non-steroidal anti-inflammatory drugs (NSAIDs), calcitonin, opioids, analgesics, sympatholytic agents and sympathetic nerve and ganglion blockade, bisphosphonate and physical therapy), the natural history of post-traumatic bone contusions has been poorly investigated, especially at long-term [5-7]. The evolution of CRPS is influenced by several factors, but pain and functional loss may last for a long time and even for a whole life.

Teriparatide (TPT) is a recombinant synthetic version of the human parathyroid hormone. Officially indicated for the treatment of postmenopausal or glucocorticoids-induced osteoporosis, TPT may also increase bone mass in men with primary or hypogonadal osteoporosis, directly enhancing bone formation and quality, thus significantly reducing the risk of secondary fracture [8]. A first off-label use of TPT has been investigated for the treatment of aseptic bone non-unions with a good safety profile and promising preliminary result [9]. Moreover, it has been successfully applied in case of transient osteoporosis and osteonecrosis of the hip [10,11]. Among the causes of BME at the knee joint, those secondary to CRPS are less investigated, and limited treatment options are available up to date.

We present two cases of chronic sBME of the knee secondary to CRPS successfully treated with TPT with complete symptoms improvement and full recovery of the function.

\section{Case Presentation}

\section{First case}

A 44-year-old man attended our outpatients' clinic for persistent right knee swelling after direct trauma occurred three months earlier. MRI, performed in the emergency room, showed extensive edema of the trabecular bone of the medial femoral condyle, without fractures lines. The patient was discharged with a plaster immobilization of the knee, protected weight-bearing and oral NSAIDs. During the immobilization 


\section{Cureus}

period, the patient reported an increase in pain and paresthesia of the knee. At the first follow-up evaluation, the patient presented significant joint effusion and limited knee range of motion (ROM) without signs of knee instability. On the anteromedial aspect of the knee, the skin was atrophic and discolored, cold and with a peculiar area of allodynia. Under the impression of post-traumatic CRPS, MRI scan was performed to detect possible trabecular fractures, showing a large area of edema of the medial femoral condyle which was diagnosed as an algodystrophic modification (Figure 1).

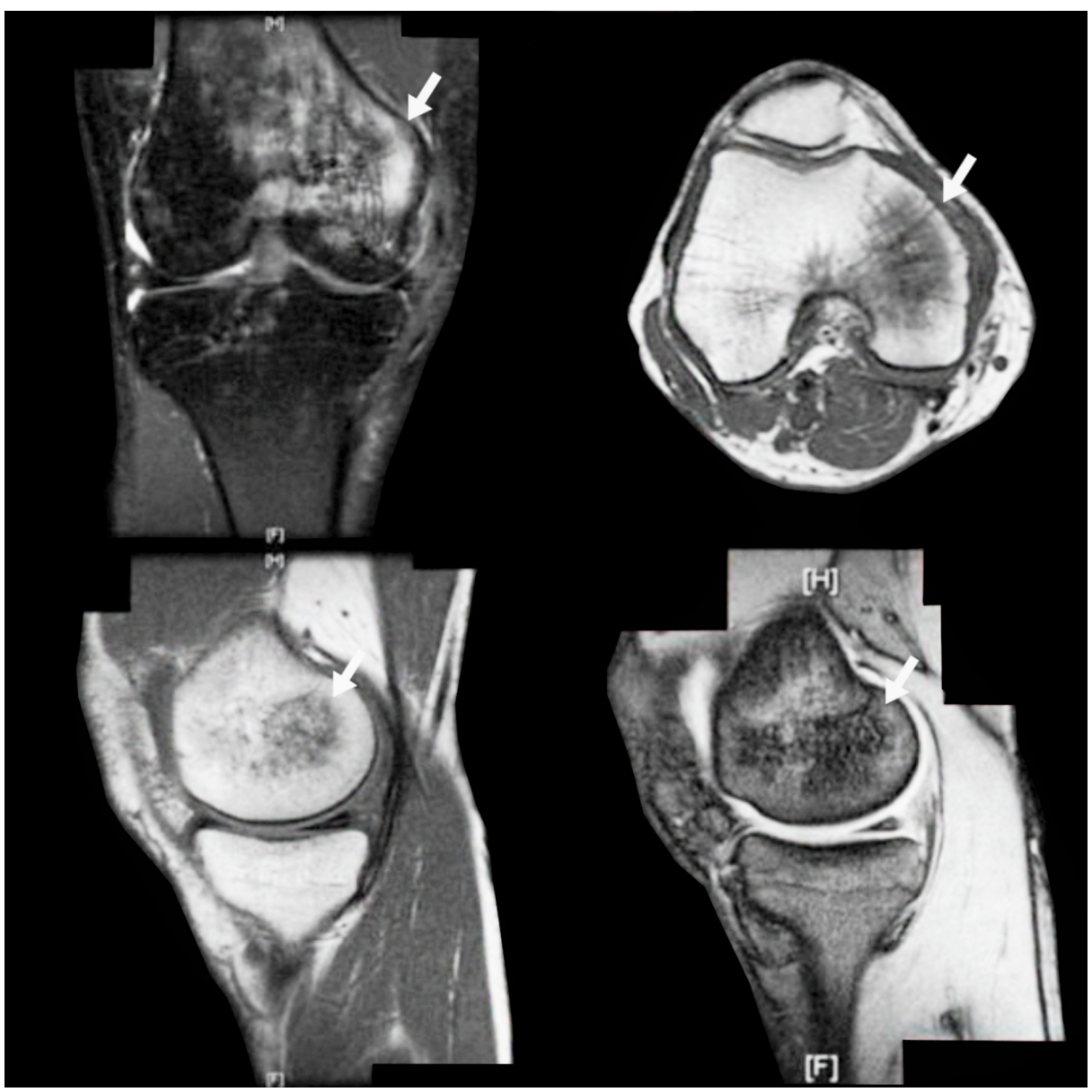

FIGURE 1: First case before teriparatide (TPT) treatment

The arrows show area of bone edema.

All diagnostic tests were performed to rule out other possible causes. A combined treatment with low dose oral prednisone, gabapentin $300 \mathrm{mg}$ three times a day, daily calcium carbonate $1250 \mathrm{mg}$-cholecalciferol 400 UI and intramuscular clodronate $200 \mathrm{mg}$ daily for 15 days was prescribed. Additionally, a rehabilitation program was initiated. The treatment was discontinued after four months due to the lack of any symptom's improvement. Afterward, the patient underwent TPT (20 mcg day) and calcium carbonate/cholecalciferol (1250 mg/400 UI) supplementation. After one month, the patient reported a marked subjective improvement in terms of both pain and joint mobility. On clinical examination, no joint swelling was noticed, with almost complete recovery of range of motion and disappearance of the cold skin overlying the medial femoral condyle. The patient started physical training and the knee function was completely recovered after one month. After three months, MRI showed almost complete resolution of the joint effusion and bone edema (Figure 2). 


\section{Cureus}

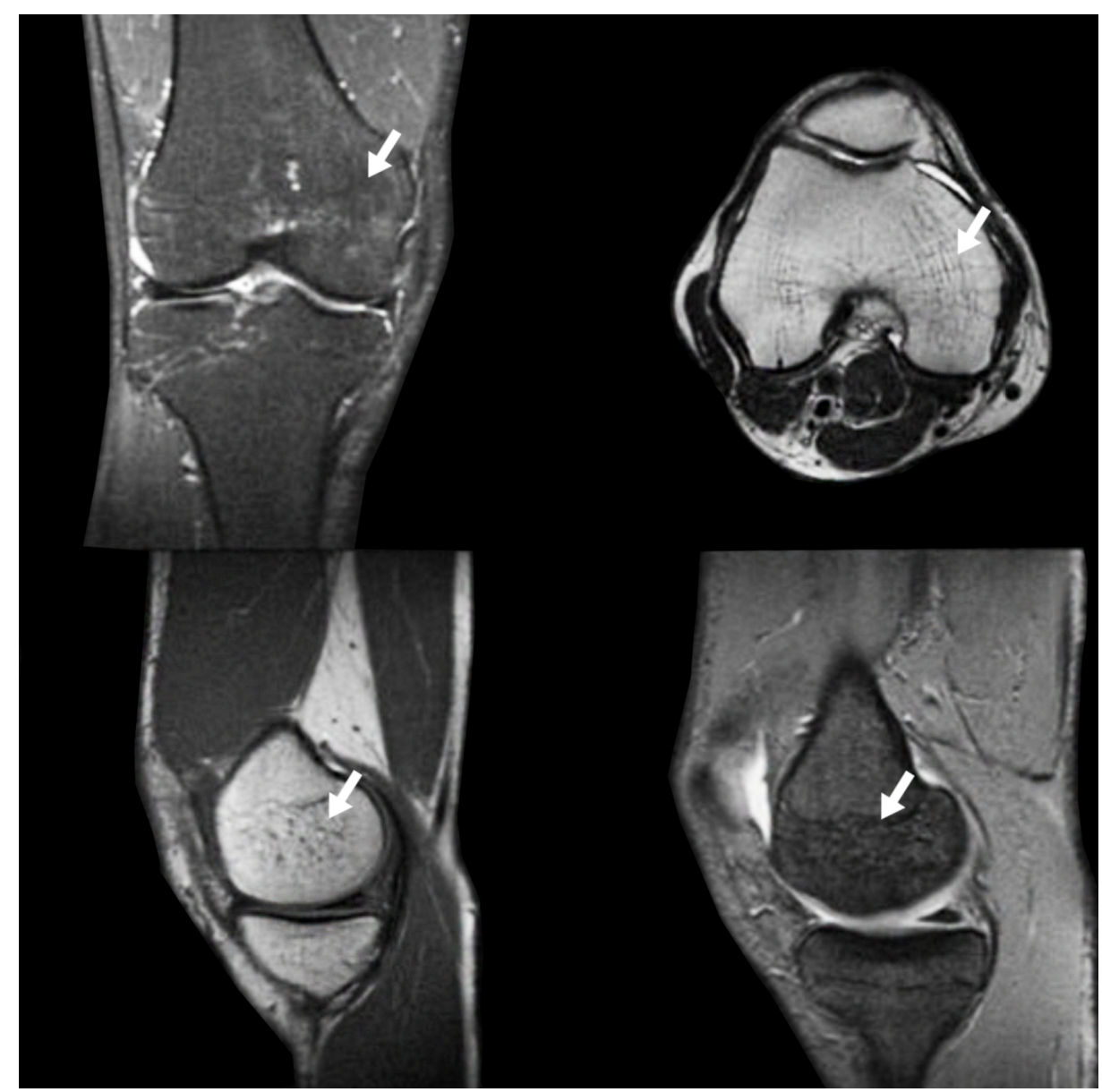

FIGURE 2: First case after teriparatide (TPT) treatment

The arrows show resolution of the bone edema.

TPT was then discontinued, but the rehabilitation program was maintained. At two years of follow-up, no recurrence of joint swelling and pain was reported, and the patient was back to regular sports and work activities.

\section{Second case}

A 21-year-old football player was sent from the orthopedic clinic for persistent knee pain and swelling, started after trauma six months before. Neither prior history nor trauma or joint disease was detected. MRI showed joint effusion and osteochondral sufferance of the lateral femoral condyle. The patient was given etoricoxib $(90 \mathrm{mg})$, glucosamine $(1500 \mathrm{mg})$ and methylsulfonylmethane $(5 \mathrm{gr} / \mathrm{daily})$ at first, but a subjective worsening of symptoms was reported, together with onset of allodynia, hyperalgesia, and progressive loss of joint function. CRPS was suspected, and alendronate $70 \mathrm{mg} /$ weekly and calcium carbonate - cholecalciferol supplement (1250 mg/400 UI) were prescribed but no improvement was noted. In fact, at the first evaluation, knee joint effusion with limited range of motion was present with difficulty to stand on the affected limb with area of allodynia and hypotrichosis on the knee. MRI showed diffuse progressive trabecular bone edema and bone resorption (Figure 3). 


\section{Cureus}

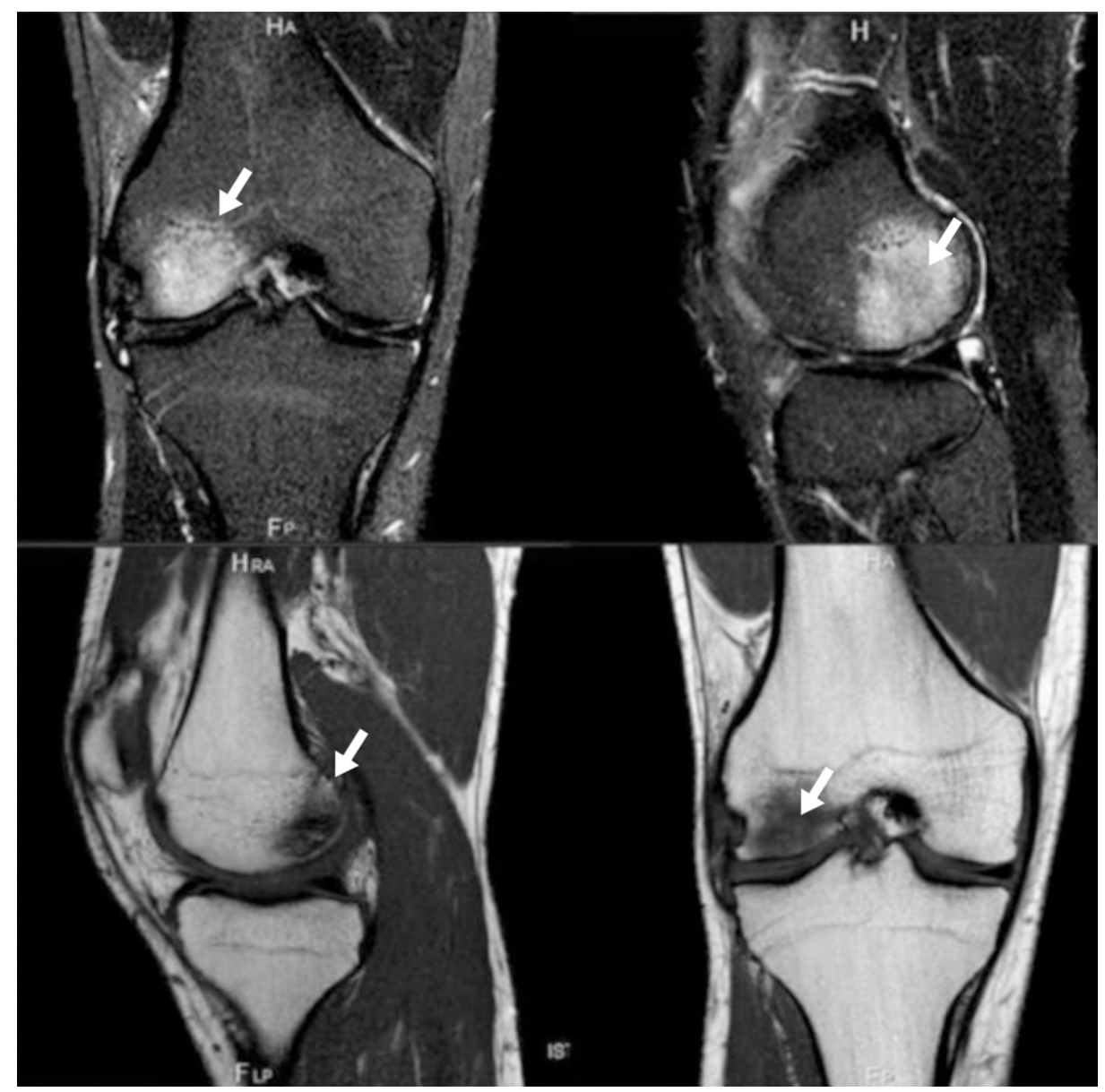

FIGURE 3: Second case before teriparatide (TPT) treatment

The arrows show area of bone edema.

All laboratory tests (erythrocyte sedimentation rate, C-Reactive protein, parathormone, bone alkaline phosphatase, serum and urinary calcium/phosphate and 25(OH)D3) were normal. The patient later received daily TPT $20 \mathrm{mg}$ daily for three months. A rehabilitation program was given to recover ROM and muscles tone of the affected knee. After the first month, only residual minimal effusion and muscle hypotrophy were presented and the patient was able to start strength training without suffering consequence. After three months, no joint effusion was detectable and complete recovery of knee function and muscles mass were evident. Control MRI showed trabecular bone edema nearly disappeared (Figure 4). 


\section{Cureus}

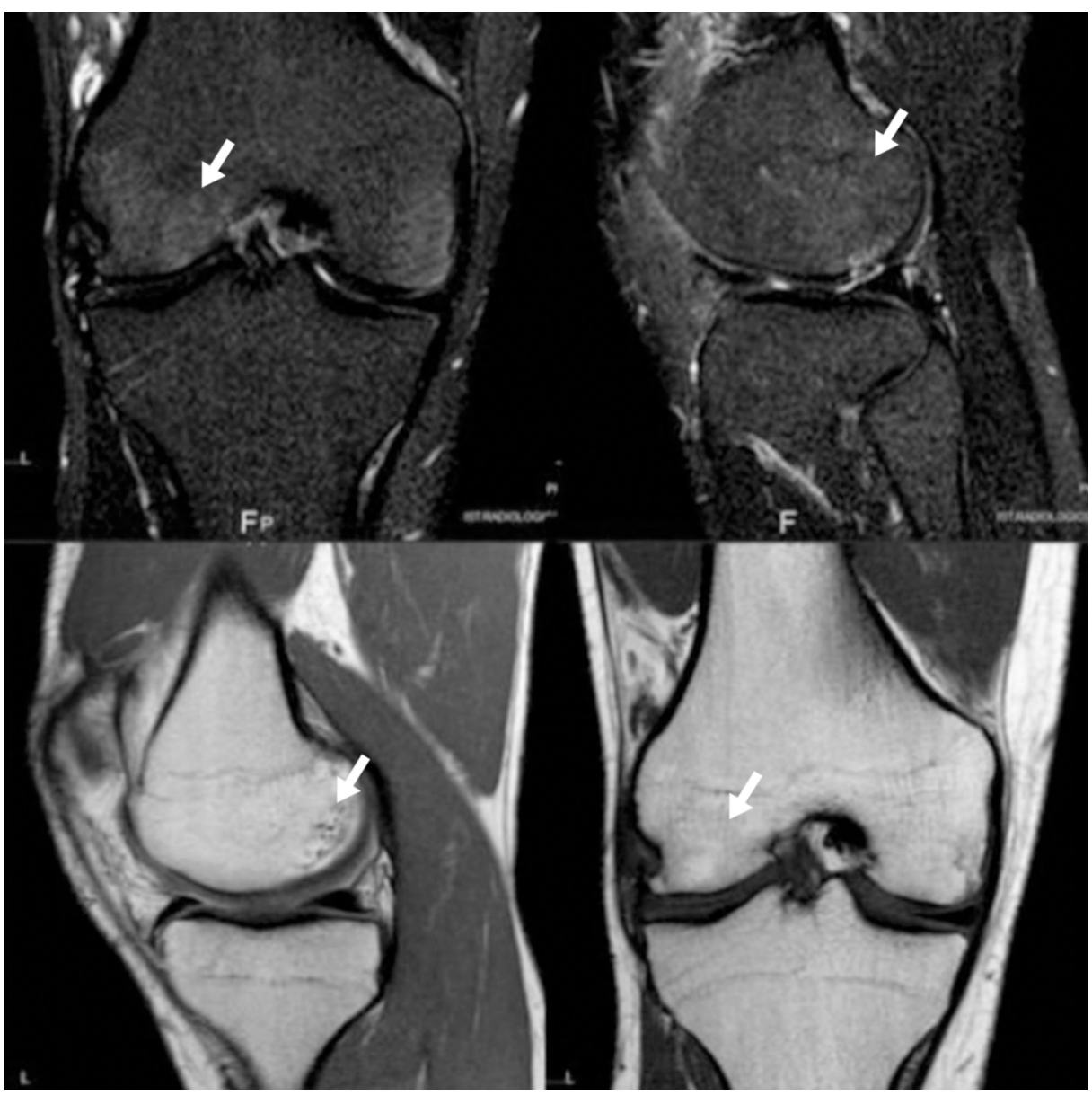

FIGURE 4: Second case after teriparatide (TPT) treatment

The arrows show resolution of bone edema.

TPT was then discontinued. Neither side effects nor symptoms recurrence during and after discontinuation of treatment was experienced. At two years of follow-up, the patient can still conduct regular competitive sport activities.

\section{Discussion}

Despite the increased scientific interest and numerous publications in the literature, the pathophysiology of sBME and CRPS is still not clarified. In fact, CRPS includes a variety of pathological processes ranging from central and peripheral sensitization, sympathetic dysfunction, abnormal inflammation with overproduction of proinflammatory cytokines and neuropeptides, enhanced bone resorption and turnover to aberrant healing. The clinical picture and the symptoms are often highly variable across cases, especially for those after trauma the treatment strategy is still challenging for most physicians. Thus, the choice of the most suitable therapy depends individual experience and expertise. In our report, the patients were initially treated with NSAIDS, bisphosphonate (clodronate and alendronate) and joint immobilization but the treatment failure led us to try TPT for a short-term period (three months). The rationale of TPT use is based on the regulatory function of parathormone. In fact, TPT is a recombinant biosynthetic version of the Nterminal of human PTH, is an anabolic agent directly stimulating bone formation and improving bone mass and quality [12]. It has been demonstrated that the maximum anabolic effect is obtained in the first period of treatment while bone remodeling ensues [13]. Other factors, like the up-regulation of growth factors including basic-fibroblast growth factor-2 (bFGF-2) and insulin-like growth factor-1 (IGF-1), receptor activator of nuclear factor-kappa B ligand (RANKL), the influence on the Wnt/ $\beta$-catenin signaling pathway and finally the transcriptionally suppression of the sclerostin gene may play an important role on the anabolic and regulating effect of TPT on bone [14-16]. As previously reported, TPT has an adrenal effect leading to an increase of plasma and urinary cortisol during the treatment period [17]. The importance of this effect is still unclear but it may have a regulatory effect on the inflammation cascade. Many studies have shown that TPT accelerated fracture healing and has also a potential effect in increasing bone volume within joints and inhibiting articular cartilage degeneration [18-20].

TPT may play a key role in patients with BME secondary to CRPS refractory to standard treatments regarding 
its sustained effect in pain reduction and knee functional improvement. The rapid response to TPT and the possibility to discontinue it after only three months may be due to not only in its anabolic properties, but also to the ability to regulate the "bone system" including cell signaling and network.

One limitation of this case reports is that we cannot exclude the influence of previous therapy or a spontaneous resolution of the disease. However, the immediate improvement after starting TPT compared to the ineffectiveness of the previous drugs could be considered a direct effect. In refractory cases of BME secondary to CRPS, as well as seen for hip osteonecrosis, TPT might be considered as an alternative treatment, although further confirmation is needed [11].

\section{Conclusions}

The rapid and sustained pain reduction with recovery of knee function for two years following administration of TPT demonstrated its potential in treatments of bone edema due to complex regional pain syndrome.

\section{Additional Information \\ Disclosures}

Human subjects: Consent was obtained by all participants in this study. Ethics Committee issued approval Not applicable. Ethics and consent to participate. Approval of the local ethics committee is not required for this treatment. Patients have signed informed consent to treatment. Consent for publication. Patients have granted their consent to the publication of the results. Conflicts of interest: In compliance with the ICMJE uniform disclosure form, all authors declare the following: Payment/services info: All authors have declared that no financial support was received from any organization for the submitted work. Financial relationships: All authors have declared that they have no financial relationships at present or within the previous three years with any organizations that might have an interest in the submitted work. Other relationships: All authors have declared that there are no other relationships or activities that could appear to have influenced the submitted work.

\section{References}

1. Baumbach SF, Pfahler V, Bechtold-Dalla Pozza S, et al.: How we manage bone marrow edema-An interdisciplinary approach. J Clin Med. 2020, 9:551. 10.3390/jcm9020551

2. Classification of chronic pain. Descriptions of chronic pain syndromes and definitions of pain terms. Prepared by the International Association for the Study of Pain, Subcommittee on Taxonomy. Pain Suppl. 1986, 3:1-226.

3. Veldman PHJM, Reynen HM, Arntz IE, Goris RJA: Signs and symptoms of reflex sympathetic dystrophy: prospective study of 829 patients. Lancet. 1993, 342:1012-1016. 10.1016/0140-6736(93)92877-V

4. Harden RN, Bruehl S, Perez RS, et al.: Validation of proposed diagnostic criteria (the "Budapest Criteria") for Complex Regional Pain Syndrome. Pain. 2010, 150:268-274. 10.1016/j.pain.2010.04.030

5. Varenna M, Adami S, Rossini M, et al.: Treatment of complex regional pain syndrome type I with neridronate: a randomized, double-blind, placebo-controlled study. Rheumatology (Oxford). 2013, 52:534542. 10.1093/rheumatology/kes312

6. Sharma A, Williams K, Raja SN: Advances in treatment of complex regional pain syndrome: recent insights on a perplexing disease. Curr Opin Anaesthesiol. 2006, 19:566-572. 10.1097/01.aco.0000245286.30282.ab

7. O’Connell NE, Wand BM, McAuley J, Marston L, Moseley GL: Interventions for treating pain and disability in adults with complex regional pain syndrome - an overview of systematic reviews. Cochrane Database Syst Rev. 2013, 2013:CD009416. 10.1002/14651858.CD009416.pub2

8. Blick SKA, Dhillon S, Keam SJ: Teriparatide: a review of its use in osteoporosis . Drugs. 2008, 68:2709-2737. 10.2165/0003495-200868180-00012

9. Canintika AF, Dilogo IH: Teriparatide for treating delayed union and nonunion: a systematic review . J Clin Orthop Trauma. 2020, 11:S107-S112. 10.1016/j.jcot.2019.10.009

10. Asadipooya K, Graves L, Greene LW: Transient osteoporosis of the hip: review of the literature . Osteoporos Int. 2017, 28:1805-1816. 10.1007/s00198-017-3952-0

11. Galluccio F, Matucci-Cerinic M: Efficacy of short-term teriparatide for hip osteonecrosis . J Rheumatol. 2016, 43:2084-2085. 10.3899/jrheum.160190

12. Dhillon RS, Schwarz EM: Teriparatide therapy as an adjuvant for tissue engineering and integration of biomaterials. J Mater Res. 2011, 4:1117-1131. 10.3390/ma4061117

13. Rubin MR, Bilezikian JP: The anabolic effects of parathyroid hormone therapy. Clin Geriatr Med. 2003, 19:415-432. 10.1016/s0749-0690(02)00074-5

14. Hurley M, Yao W, Lane NE: Changes in serum fibroblast growth factor 2 in patients with glucocorticoidinduced osteoporosis treated with human parathyroid hormone (1-34). Osteoporos Int. 2005, 16:2080-2084. 10.1007/s00198-005-1998-x

15. Rosen CJ: The role of parathyroid hormone in the management of osteoporosis . Horm Res. 2005, 64:81-85. 10.1159/000087762

16. Paszty C, Turner CH, Robinson MK: Sclerostin: a gem from the genome leads to bone-building antibodies . J Bone Mineral Res. 2010, 25:1897-1904. 10.1002/jbmr.161

17. Lasco A, Catalano A, Morabito N, Gaudio A, Basile G, Trifiletti A, Atteritano M: Adrenal effects of teriparatide in the treatment of severe postmenopausal osteoporosis. Osteoporos Int. 2011, 22:299-303. 10.1007/s00198-010-1222-5 


\section{Cureus}

18. Kakar S, Einhorn TA, Vora S, et al.: Enhanced chondrogenesis and Wnt signaling in PTH-treated fractures . J Bone Miner Res. 2007, 22:1903-1912. 10.1359/jbmr.070724

19. Gomberg SJ, Wustrack RL, Napoli N, Arnaud CD, Black DM: Teriparatide, vitamin D, and calcium healed bilateral subtrochanteric stress fractures in a postmenopausal woman with a 13-year history of continuous alendronate therapy. J Clin Endocrinol Metab. 2011, 96:1627-1632. 10.1210/jc.2010-2520

20. Sampson ER, Hilton MJ, Tian Y, et al.: Teriparatide as a chondroregenerative therapy for injury-induced osteoarthritis. Sci Transl Med. 2011, 3:101ra93. 10.1126/scitranslmed.3002214 